\title{
El aprendizaje en la educación superior
}

\author{
César Carbache Mora, Universidad Lacia “Eloy Alfaro” de Manabí, Ecuador
}

\begin{abstract}
Resumen: El siguiente análisis pretende investigar el presente y el futuro de la universidad ecuatoriana: su vinculación al trabajo, la ciudadanía y la identidad, la enseñanza superior y el aprendizaje, ecologías del conocimiento en cuanto a enlazar la investigación y la docencia en la educación superior, así como el pre servicio de la educación: las políticas, programas y prácticas. La sociedad de la información y el conocimiento requiere de ciudadanos mejor preparados y más comprometidos con el medio en que les toca vivir y desenvolverse. Consciente de esta realidad la universidad debe de replantearse los postulados que la llevaron a su creación. Pasar de la teorización (conceptualización - observación) a la practicidad (investigación - reflexión - criticidad-involucramiento) valorando y respetando la diversidad y la multiculturalidad a la par de concienciar en la responsabilidad social de sus conocimientos. En este replanteo la universidad de hoy debe de analizar, como lo proponen algunos investigadores (Montero, Patricio, 2010) las megas tendencias educativas como desafios para la educación universitaria que tienen implicaciones en las decisiones educacionales claves vinculadas a la pertinencia y validez de la educación superior. En especial, para el desarrollo curricular, las metodologías tanto para la enseñanza como para la evaluación y para la gestión de la docencia. Cinco son las que se plantean: 1. - La globalización marcada por la sociedad del conocimiento. 2.- Formación para una estructura laboral altamente dinámica e interdependiente. 3.- La supervivencia de las organizaciones depende centralmente del conocimiento que posee su personal. 4.- Cambios en la concepción de comportamientos inteligentes y la productividad de las personas. 5.- Cambios sobre la concepción del aprendizaje, enfatizando la preocupación de los procesos internos de las personas para el aprendizaje. ${ }^{1}$
\end{abstract}

Palabras clave: aprendizaje, sociedad del conocimiento en la información, formación continua.

\begin{abstract}
The following analysis aims to investigate the present and the future of the Ecuadorian university, its relationship to work, citizenship and identity, higher education and learning, knowledge ecologies to link research and teaching in higher education and the pre service education: policies, programs and practices. The information society and knowledge required of citizens better prepared and more committed to the environment in which they live and develop. Aware of this reality, the university must rethink the principles that led to its creation. Moving from theorizing (conceptualization - observation) to the practical (research - reflection - criticality - involvement) valuing and respecting diversity and multiculturalism in tandem to raise awareness on the social responsibility of their knowledge. This rethinking of contemporary university must analyse as proposed by some researchers (Montero, Patricio. 2010) the megas educational trends and challenges for higher education that have implications on key educational decisions related to the relevance and validity of higher education. In particular, for curriculum development, methodologies for both teaching and assessing also the management of teaching. 1.- Globalization marked by the knowledge society. 2.- Training for a highly dynamic interdependent labour structure. 3.- The survival of organizations depends on the knowledge possessed by its staff. 4.- Changes in the design of intelligent behaviour and productivity of people. 5.- Changes on the conception of learning, emphasizing the concern of internal processes of people for learning.
\end{abstract}

Keywords: Learning, Knowledge Society in Information, Training.

\section{Introducción}

$\mathrm{L}$ a educación terciaria o post secundaria en Ecuador y en especial en los países en vías de desarrollo de América Latina, viene atravesando una especie de transición, saltos entre los modelos de desarrollo -industrialización (modernismo) y sociedad de la información (post modernismo). A pesar de que la económica ancestral de estos países sea la agricultura, la ganadería y la pesca. Quedando como punto a parte la explotación petrolera que significa, en algunos casos, como lo es en la ecuatoriana, el primer rubro en la economía.

Este panorama (tecnológico-productivo), a su vez, ha generado cambios en todos los contextos educativos y la educación superior no es una excepción. Proceso que en la sociedad ecuatoriana y en

\footnotetext{
${ }^{1}$ Montero, P. (2010). Megatendencias educativas del siglo XX y prospectiva para el siglo XXI: desafíos para la educación universitaria. Santiago, Chile: Universidad de Santiago de Chile.
}

Revista Internacional de Aprendizaje en la Educación Superior

Volumen 2, Número 2, 2015, <http://sobrelaeducacion.com>, ISSN 2386-7582

(C) Global Knowledge Academics. César Carbache Mora.

Todos los derechos reservados. Permisos: soporte@gkacademics.com 
el resto de América Latina, avanza de manera lenta, por las realidades internas de cada país: como el analfabetismo (que de acuerdo a la Organización de Estados Iberoamericanos) OEI, es la gran deuda social y educativa que afecta a más de 40 millones de personas adultas -encuestadas en el 2000que a nivel de 19 países de América Latina, no pueden acceder, elegir y apropiarse de las oportunidades que ofrece la vida en sociedad. ${ }^{2}$

El informe de la Comisión Económica para América Latina y el Caribe (CEPAL) 2013, al referirse a la pobreza y extrema pobreza, indica que en el 2012 un 28,2\% de la población de América Latina era pobre y la indigencia, o pobreza extrema, llegaba a un 11,3\%. Esto significa que 164 millones de personas eran pobres, de las cuales 66 millones se ubicaron en los pobres extremos. ${ }^{3}$

El desempleo y el subempleo, analizados por la CEPAL y la Organización Internacional del Trabajo (OIT) 2013, señalan que la tasa de desempleo de América Latina y el Caribe en 2012 fue la más baja de las últimas décadas, tras descender al 6,4\%, del 6,7\% que registró el año anterior. Esta cifra es especialmente relevante a la luz de la difícil situación que impera en los mercados de trabajo de otras regiones del mundo. Los indicadores laborales mejoraron pese al modesto crecimiento de la economía regional, de apenas un 3,0\%. A pesar de un marcado aumento de la participación laboral, el número de desempleados urbanos bajó aproximadamente 400.000 personas, gracias a una generación de empleo relativamente fuerte. Sin embargo, aún quedan alrededor de 15 millones de personas desocupadas que buscan trabajo. ${ }^{4}$

Los cierto es que para mejorar los estilos de vida de las personas de los estratos medios y bajos de nuestras poblaciones, señala el documento, habrá necesidad de una fuerza laboral con habilidades, competencias y conocimientos que se ajusten a las necesidad de las empresas, lo que exige a la vez una buena red de sistemas de formación profesional y capacitación. En suma, mejorar los sistemas educativos permitiendo un mayor acceso a las universidades provocando una sinergia entre la propuesta universitaria y el segmento laboral.

Entonces, ¿Cuál debería ser el nuevo perfil de la educación superior? ¿Cómo articular la universidad al sector productivo? ¿Cómo mejorar las políticas de acceso a la educación post secundaria? ¿Con qué elementos deben de construirse los nuevos currículos para la enseñanza aprendizaje de los nuevos ciudadanos? ¿Sin recursos en las universidades, el axioma de la investigación es posible?

Sin duda hay más preguntas que respuestas para dilucidar este paradigma, en estos tiempos donde el capital y la tierra han dejado sus viejos postulados a la información y el conocimiento, como razón de ser social y económica.

\section{El futuro de la universidad ecuatoriana: su vinculación al trabajo, la ciudadanía y la identidad}

Desde la perspectiva académica, el tema de fondo es que la universidad ecuatoriana, bien sea pública o privada, tiene que reorientarse, si es que quiere constituirse en ejemplar y "competitiva" en el ámbito internacional. Entre los más importantes índices mundiales sobre los niveles superiores no se halla una sola universidad ecuatoriana, pero sí se encuentran algunas latinoamericanas. La excepción es la Escuela Superior Politécnica del Litoral, considerada en alguno de aquellos "rankings".

Las universidades ecuatorianas se han organizado para dar clases y producir profesionales. En casi todas se evalúa "la clase" y al profesor. Si, además, el profesor realiza investigaciones y tiene renombre académico, también la universidad se prestigia. Pero el espacio investigativo es marginal. No forma el centro del sistema.

A ello se suma el burocratismo creciente y su influencia administrativa en la universidad. Me ha sorprendido cómo en diversas instituciones las "mediciones de calidad" y los sistemas "ISO" se han introducido en perjuicio del mundo académico. Desde luego, de allí derivan los "controles de procesos" que llegan hasta las aulas y la presencia del profesorado. Y éste no es solo un problema nacional. ${ }^{5}$

\footnotetext{
${ }^{2}$ Organización de Estados Iberoamericanos. (2013). El analfabetismo en América Latina. Buenos Aires, Argentina: SITEAL.

${ }^{3}$ Comisión Económica para América Latina y el Caribe. (2014). Panorama Social de América Latina. Santiago, Chile: CEPAL.

${ }^{4}$ Comisión Económica para América Latina y el Caribe. (2013). Coyuntura laboral en América Latina y el Caribe. Informe CEPAL/OIT. Santiago, Chile: CEPAL.

${ }^{5}$ Paz y Miño, J. (2008). Universidad ecuatoriana ¿hacia el futuro? Quito, Ecuador: Pontificia Universidad Católica del Ecuador.
} 
Pérez Gómez, pedagogo español, 2010, en el artículo "Nuevas exigencias y escenarios para la profesión docente en la era de la información y de la incertidumbre", alude a que el desafío actual más urgente de nuestro sistema educativo es preparar a los ciudadanos para afrontar la cambiante, incierta, compleja y profundamente desigual sociedad contemporánea en la era de la información y de la incertidumbre. Los contextos y escenarios sociales que rodean la vida de las nuevas generaciones en nada se parecen a los escenarios y contextos que rodeaban el crecimiento de las generaciones del siglo XIX y primera mitad del siglo XX.

Sin embargo, el dispositivo escolar vigente, el curriculum escolar organizado en disciplinas, la forma habitual de organizar el espacio y el tiempo, los modos de agrupar a los estudiantes, los métodos de enseñanza, los sistemas de evaluación y calificación del alumnado, y los sistemas, programas e instituciones de formación de docentes son esencialmente los mismos que se establecieron ya en el siglo XIX y que, con modificaciones cosméticas, se han mantenido y reproducido hasta nuestros días.

En el centro del cambio cualitativo requerido se sitúa la figura de un nuevo docente como profesional capaz de comprender la complejidad y la incertidumbre de la era contemporánea y comprometido con la misión de acompañar, orientar y estimular el desarrollo y aprendizaje relevante de cada uno de los estudiantes a su cargo.

La formación de estos docentes requiere un cambio sustancial en los programas, métodos e instituciones en todas las etapas de su larga vida profesional. Parece fundamental que los docentes vivan, trabajen, analicen y evalúen las posibilidades educativas de diferentes proyectos, experiencias, contextos y situaciones escolares al mismo tiempo que tienen que responder a las exigencias y tensiones de dichos escenarios. ${ }^{6}$

La mundialización y globalización son fenómenos que están asociados a la globalización financiera estimulada por el desarrollo de las tecnologías de la información y la comunicación. Ésta ha generado una suerte de nuevo contexto mundial donde lo económico, como un proceso pluridimensional, arrastra a otros aspectos vinculados a las finanzas, las ciencias, las tecnologías, las comunicaciones, la educación, la cultura, la política, entre otros estamentos.

$\mathrm{Y}$ con ello se señalan las nuevas tendencias que exigen nuevos requerimientos para la formación de una generación telemática y tecnológica. Lo que quiere decir, la reelaboración de un currículum que no solamente se enfoque en la enseñanza (currículo de contenidos) o el aprendizaje (currículo de aprendizaje) sino que, además, de esos logros de aprendizaje, se consiga que ese futuro profesional desarrolle holísticamente y con autonomía sus saberes (currículo por competencias); con lo que se debería de construir un ciudadano vinculado a la ecología del conocimiento, con responsabilidad social y autosuficiente frente a la incertidumbre del entorno y sus contextos.

Dentro del proyecto educativo diseñado por la Universidad Internacional SEK Ecuador, denominado "Modelo educativo basado en competencias de formación integral", se señala que la importancia de considerar el enfoque de las competencias en la educación superior radica en primer lugar, en el aumento de la pertinencia de los programas con relación a la demanda del contexto social en su conjunto, involucrando lo profesional y lo organizacional. En segundo lugar, la posibilidad de gestionar la calidad de los procesos de aprendizaje de los estudiantes, considerando dos elementos centrales: la evaluación de la calidad del desempeño y la evaluación de la formación que ofrece la institución formadora. En tercer lugar, la formación por competencias está alcanzando el nivel de una política educativa internacional, a la cual están contribuyendo los aportes conceptuales y metodológicos de las investigaciones desarrolladas en los últimos años.

Existen muchas definiciones respecto a lo que se debe entender como competencias, la Comisión Europea en el 2004 definió como competencia, "Una combinación de destrezas, conocimientos, aptitudes y actitudes, y a la inclusión de la disposición para aprender, además del saber cómo. (...) Las competencias clave representan un paquete multifuncional y transferible de conocimientos, destrezas y actitudes que todos los individuos necesitan para su realización y desarrollo personal, inclusión y empleo. Éstas deberían haber sido desarrolladas para el final de la enseñanza o forma-

\footnotetext{
${ }^{6}$ Pérez, A. (Coord.). (2010). Nuevas exigencias y escenarios para la profesión docente en la era de la información y la incertidumbre. Málaga, España: Universidad de Málaga.
} 
ción obligatoria, y deberían actuar como la base para un posterior aprendizaje como parte de un aprendizaje a lo largo de la vida."7

Un documento elaborado por el ministerio de educación de El Salvador, titulado "¿Qué aprender? ¿Qué enseñar? ¿Cuándo enseñar? ¿Cómo enseñar?, currículo al servicio del aprendizaje”, 2008, señala que el aprendizaje por competencias está orientando el currículo al desarrollo de competencias para que se concreten en los objetivos, contenidos, metodología, recursos y evaluación con mayor articulación y claridad. Es en esta instancia donde la validez del constructivismo posibilita el desarrollo de competencias, ya que este enfoque promueve que el alumno abandone su actitud de receptor pasivo, para convertirse en un activo protagonista de su propio aprendizaje. ${ }^{8}$

Según Pierre Astolfi, el aprender comprende tres etapas: información, conocimiento y saber, por las que debe transitar aquel que alcance el aprendizaje profundo. Las instituciones educacionales tienen así la tarea de organizar sus procesos de enseñanza y aprendizaje inter y extra aula, considerando estas etapas didácticas. En el fondo de ellas, está el desarrollo de las habilidades intelectuales de los alumnos y alumnas, organizado de manera planificada y consciente para alcanzar en ellos un nivel de reflexión que les permita la autonomía intelectual necesaria para crear y creer en los fundamentos que se constituyan en la base de los continuamente renovados espacios de intervención social. ${ }^{9}$

Sin embargo, aquí surgen una serie de cuestionamientos que nos conllevan a reflexionar. ¿Cómo formar al nuevo ciudadano dentro de aulas tradicionales? Los currículos elaborados para una generación con otras necesidades de formación, ¿Serán los correctos para educar a los ciudadanos de la sociedad de la información y el conocimiento? ¿Serán suficientes las capacidades del profesorado que mantiene una matriz mental tradicionalista, donde las clases magistrales, la memorización, la evaluación sumativa y el desconocimiento tecnológico, por señalar algunas, son sus argumentos más cotidianos para educar a ese ser humano del mañana?

Hay que repensar también que la universidad de este siglo (XXI) debe ampliar su visión respecto de la sola productividad cognitiva de los ciudadanos, es decir, creadora de mentes brillantes en las tecnologías y las ciencias, sin considerar la parte afectiva-social de su formación, de compaginación con su entorno e identidad.

La vocación profesional del titulado o novel profesional no sólo debe servirle de sustento económico sino que, además, concientice sobre el retorno de sus conocimientos para mejorar su entorno social, cultural, político, económico más próximo y el de la sociedad misma.

En ese aspecto comparto el criterio de Dias Sobrinho, $2007,{ }^{10}$ que dice no hay que olvidar que la educación es un proceso humano y por tanto, lo primero que habrá que conseguir es la humanización de los profesionales, con sentido de pertinencia, responsabilidad social, posibilitados para tomar decisiones y resolver problemas en entornos complejos en todos los momentos de la vida.

El futuro de la universidad en Ecuador y en el mundo contemporáneo, debe de mantener una sintonía con su micro y macro entorno, no obstante, alimentarse de su hábitat natural: sus propias realidades, pero sin quitarle la mirada al mundo globalizado, ya que en esos estadios laborales deberán de demostrar su eficiencia y eficacia los profesionales que emerjan de esos claustros. Y es que una universidad sin calidad, afectividad e identidad no sirve ni a los estudiantes ni a la sociedad.

La vida económicamente activa precisa de profesionales con una serie de cualidades que partan desde la base cognitiva (metacognitiva) pasando por destrezas y habilidades (creación adaptación e innovación de recursos) que puedan desarrollarse, acompañadas por actitudes innovadoras en los aspectos de trabajar en equipo, empatía, iniciativa para tomar decisiones, saber comunicarse y valo-

\footnotetext{
${ }^{7}$ Universidad Internacional SEK Ecuador. (2011). Modelo educativo basado en competencias de formación integral. Quito, Ecuador: UISEK.

${ }^{8}$ Ministerio de Educación de El Salvador. (2008). Currículo al servicio del aprendizaje. Aprendizaje por competencias. San Salvador, El Salvador: Ministerio de Educación.

${ }^{9}$ Mella, E. (2003). La educación en la sociedad del conocimiento y del riesgo. Revista enfoques educacionales, 5(1). Universidad de Magallanes.

${ }^{10}$ Días, J. (2007). Calidad, pertinencia y responsabilidad social de la universidad latinoamericana y caribeña. En A. L. Gazzola y A. Didriksson (Eds.), Tendencias de la educación superior en América Latina y el Caribe (pp. 87-112). Caracas, Venezuela: UNESCO IESALT.
} 
res como la responsabilidad, el respeto a la diversidad y el deseo de superación. Lo que se conoce como competencias básicas.

Steven Johnson. 2011, en su libro "Las buenas ideas: una historia natural de la innovación", destaca que hoy en día las innovaciones son resultados de redes que evolucionan por medio de procesos colectivos y distribuidos en un gran número de grupos que trabajan en un mismo problema. Destacando que este proceso de innovación ha sido posible gracias a una añeja actividad como es la cultura del café, donde investigadores, filósofos, académicos, empresarios pueden compartir ideas y promoverlas.

Desde esta perspectiva la universidad tanto ecuatoriana como latinoamericana, debe de alejarse de esa visión contemplativa (teórica-observadora) para articularse con la dinámica del tiempo y la realidad de cada sociedad (activa-involucrada-investigativa-generadora de nuevos conocimientos) en beneficio de los contextos actuales y por ende de las personas.

Por ello vincular universidad y trabajo es otro de los retos en que las instituciones de educación superior IES en Ecuador y América Latina deben de emprender tan prontamente como les sea posible, aunque esto signifique adecuar, adaptar, reformular y crear nuevos contextos (currículos) con enfoques de pertinencia, buscando desarrollar los saberes en procura siempre de la autonomía del aprendiz.

Además, de la generación de políticas de estado en aspectos sociales y educacionales. Recursos para la implementación de las nuevas directrices: tecnología de punta, infraestructura, recursos para proyectos de investigación, reeducación (estudios de post grados) articulados a las nuevas tendencias que se desarrollan en el campo laboral.

Empero este particular objetivo no es tan fácil de alcanzar si consideramos que los pueblos como Ecuador y otros en latinoamericana mantienen situaciones precarias de desarrollo, temas tratados con anterioridad. Lo que genera una suerte de deserción escolar en todos los niveles educativos y la Universidad no es la excepción. Los padres prefieren hijos trabajando, procurando recursos para calmar las necesidades básicas, que hijos estudiando. El tema de la educación tiene una problemática profunda, que no es muy fácil de obviar o pasar por alto.

De acuerdo al informe de la OEI, del “¿por qué los adolescentes dejan la escuela?”, menciona que durante la última década se intensificó el proceso de expansión de la escolarización de niños y adolescentes. La última información disponible indica que más del 97\% de los niños de entre 7 y 12 años y más del $83 \%$ de los adolescentes de entre 13 y 17 años concurren a la escuela. Puestos en perspectiva, estos valores revelan un incremento en la proporción de niños y adolescentes escolarizados en dos y seis puntos porcentuales respectivamente durante el período 2000-2010. ${ }^{11}$

En el Ecuador la tasa de no participación de los bachilleres en la educación post secundaria por razones económicas fue en el 2007 del $45 \%$ mientras que para el 2011 fue del 25\%, de acuerdo a la Secretaría Nacional de Educación Superior Ciencia y Tecnología SENESCYT.

\section{La enseñanza superior y el aprendizaje}

Parecería contradictorio pensar que la educación terciaria o post secundaria se sigue practicando bajo conceptos, estructuras, criterios y axiomas de hace más de un siglo. A pesar de que la evolución humana y por consiguiente científica, tecnológica, educacional, vive y convive entre otros contextos, muy diferentes, como de la información y del conocimiento, de la telemática y la tecnología, las ciencias y los nuevos paradigmas. Es decir, en una realidad muy distinta a la realidad con que fueron formados los que ahora enseñan en los claustros universitarios.

A ello se suma la falta de la formación y actualización constante del profesorado universitario. Quienes carecen de las herramientas pedagógicas (actualmente andragógicas) y metodológicas que les permitan arribar a ese punto deseado de la educación integral u holística donde el conocimiento facilita el desarrollo de habilidades y destrezas y por tanto la concreción de los saberes (metacognitividad) en el mejoramiento de las sociedades en la que les toca vivir.

\footnotetext{
${ }^{11}$ Organización de Estados Iberoamericanos. (2013). ¿Por qué los adolescentes dejan la escuela? Buenos Aires, Argentina: SITEAL.
} 
John Elliott y Santos Guerra (...) consideran de primordial importancia el carácter cooperativo de los procesos de actualización y formación del profesorado estrechamente ligado a las situaciones, problemas y escenarios reales, así como a las prácticas concretas que cada uno desarrolla. Ambos también, siguiendo la estela de Stenhouse, conceden una importancia decisiva a la experimentación del curriculum como la mejor estrategia para provocar el desarrollo de la autonomía profesional de los docentes.

La formación continua del profesorado es una exigencia de la naturaleza misma de la profesión docente, vinculada al desarrollo y construcción personal del conocimiento en todos y cada uno de los individuos de cada generación y a la utilización personal y colectiva del conocimiento para construir las formas de vida y relaciones que constituyen la sociedad que deseamos. Nada está prescrito de antemano y todo ha de ser objeto de análisis, debate y acuerdos, con la conciencia clara de que lo que construimos y lo que decidimos para bien o para mal nos construye, nos potencia o nos limita. ${ }^{12}$

Aprender supone reconstruir (Pérez Gómez, 1998), reestructurar (Pozo, 2006), redescribir (Karmilov-Smith, 1992) de una manera consciente y sistemática el entramado de representaciones o significados, de comprensión y de acción, que cada individuo ha ido construyendo a lo largo de su historia personal, a propósito de sus interacciones en los escenarios cotidianos, a través de un largo proceso de socialización singular.

El oficio docente, para Contreras, se hace con uno mismo, con lo que uno es y lleva incorporado. Al enseñar, uno se expone, se enseña; no sólo enseña un saber, sino la propia relación con el saber; no sólo está allí, entre estudiantes, sino que es ante todo presencia... el saber que necesitamos para vivir (y para vivir-nos como docentes) es aquel que está unido a nosotros, que nos constituye, que hace cuerpo con nosotros, que tenemos incorporado. De este modo, el docente ha de considerarse como un creador de saber, no como un mero administrador de conocimientos.

En el artículo elaborado por el comité científico regional para América Latina y el Caribe del Foro de la UNESCO (Paris, Diciembre 2003), se menciona que una de las características de la sociedad contemporánea es el papel central del conocimiento en los procesos productivos, al punto que el calificativo más frecuente que suele dársele es el de sociedad del conocimiento. Asistimos a la emergencia de un nuevo paradigma económico-productivo en el cual el factor más importante no es ya la disponibilidad de capital, mano de obra, materias primas o energía.

Una buena parte de los cambios que propone el proceso de Bolonia han sido desarrollados con base en los resultados obtenidos por el proyecto Tuning Educational Structures in Europe (González y Wagenaar, 2003). En él se señala que "en el paradigma enseñanza-aprendizaje se está produciendo un cambio en el cual los esfuerzos educativos se centran cada vez con mayor intensidad en el individuo que aprende" (González y Wagenaar, 2003: 74).

Ello supone un desplazamiento de la educación centrada en la enseñanza hacia una educación centrada en el aprendizaje, que implica también un cambio en "el enfoque de las actividades educativas, los materiales de enseñanza y una gran variedad de situaciones didácticas, puesto que estimulan el compromiso sistemático del estudiante con la preparación individual o en grupo de temas importantes, presentaciones, feedback, etc." (González y Wagenaar, 2003: 75). ${ }^{13}$

La sociedad del conocimiento es también la sociedad del aprendizaje. Esta idea está íntimamente ligada a la comprensión de toda educación en un contexto más amplio: el aprendizaje a lo largo de toda la vida, donde el sujeto precisa ser capaz de manipular el conocimiento, de ponerlo al día, de seleccionar lo que es apropiado para un contexto específico, de aprender permanentemente, de entender lo que se aprende y, todo ello de tal forma que pueda adaptarlo a nuevas situaciones que se transforman rápidamente.

En este sentido, sin profundizar en los rasgos que caracterizan a esa nueva cultura del aprendizaje y la enseñanza, hay ciertas tendencias en la naturaleza de los saberes que la universidad gestiona que se deben considerar, ya que constituyen verdaderos retos que la sociedad del conocimiento plantea a la enseñanza y el aprendizaje universitarios. En primer lugar, el saber es cada vez más

\footnotetext{
12 Pérez, A. (Coord.). (2010). Nuevas exigencias y escenarios para la profesión docente en la era de la información y la incertidumbre. Málaga, España: Universidad de Málaga.

${ }^{13}$ De Alfonseti, N., Lillo, A., Asensi, M. J., Giménez, V. M., Lorenzo, J., Mira-Perceval, M. T., y Rico, J. R. (2007). Metodología docente y sistemas de evaluación del aprendizaje. Alicante, España: Universidad de Alicante.
} 
extenso. En segundo lugar, el conocimiento presenta una tendencia a la fragmentación y especialización y en tercer lugar, el ritmo de producción de ese conocimiento es cada vez más acelerado y, por tanto su obsolescencia también crece. ${ }^{14}$

En otras palabras, el profesor ha pasado de ser un simple transmisor del conocimiento a un líder intelectual (Pérez Gómez, 2010). Un tutor que acompaña al dicente en su formación, que utiliza los conocimientos previos del aprendiz para gestionar otros conocimientos y con ellos desarrollar habilidades, en un marco de valores e ideales adecuados con su identidad.

La sociedad del conocimiento consiste en buscar, procesar, crear, adaptar y aplicar el conocimiento de manera colaborativa y solidaria para resolver los problemas en lo local con una visión global, teniendo apoyo en las tecnologías de la información y la comunicación (Tobón, 2013a).

La multidisciplinariedad, interdisciplinariedad y transdisciplinariedad son conceptos que actualmente se manejan en la elaboración de un proyecto formativo que según Sergio Tobón Tobón (2013) es "una metodología didáctica para resolver un problema del contexto mediante actividades articuladas o complementarias, buscando que el estudiante tenga un producto relevante que no sea meramente el abordaje de un contenido y finalmente que se articulen por lo menos dos áreas, dos asignaturas o dos campos".

Para abordar los retos de la sociedad del conocimiento se ha venido construyendo de manera colaborativa un enfoque alternativo en Iberoamérica denominado la socioformación. Consiste en formar personas integrales para la sociedad del conocimiento con un sólido proyecto ético de vida, trabajo colaborativo, emprendimiento y gestión del conocimiento, con las competencias necesarias para identificar, interpretar, argumentar y resolver los problemas de su contexto con una visión global y a través de proyectos interdisciplinarios (Tobón, 2013a, 2013b). ${ }^{15}$

\section{Ecologías del conocimiento: vincular la investigación y la docencia en la educación superior}

El concepto de ecología del conocimiento no es nuevo: fue Kitaro Nishida (1870-1945), filósofo japonés, quien acuñó el término. Pör (1995) y Pör y Spivak (2000) retoman el concepto de Ecologías del Conocimiento (EC) y afirman también que el desarrollo del conocimiento organizacional se basa en redes de personas que interactúan, compartiendo sus conocimientos con apoyo de la tecnología. ${ }^{16}$

El concepto práctico de ecología del conocimiento se contempla en la interactuación de sus miembros al complementar sus saberes, con objetivos de enriquecimiento mutuo y en estrecha relación con las tecnologías de la información, la generación de nuevos conocimientos buscando la sustentabilidad del entorno que nos rodea.

En este sentido entonces, nace en el escenario la posibilidad de caracterizar la Ecología del Conocimiento "desde la interacción de sus miembros y la estrecha relación con la información, las tecnologías, la generación de conocimiento y el entorno que los rodea, "une la creación y utilización de conocimiento con aspectos sociales y de gestión de redes" (Santamaría, F: 2012). ${ }^{17}$

El muy citado listado de los saberes necesarios de Morín (1999) es, en realidad, una descripción de un ciudadano "ideal", este criterio se lo podría ligar a la ecología del conocimiento Para ello, es importante concienciar e involucrar a ese ciudadano contemporáneo en desarrollar las fortalezas del aprendizaje colaborativo, ya que este promueve y fomenta aspectos, como aprender a profundizar en los conocimientos, aprender a trabajar en equipo, a comprender al resto del grupo, respetando las otras opiniones, resolviendo los conflictos y a trabajar en colaboración con sus compañeros, obteniendo un producto final, en definitiva, aprender a aceptar a los demás a la vez, que se aceptan ellos

\footnotetext{
${ }^{14}$ Fernández, A. (2003). Nuevas metodologías docentes. Valencia, España: Universidad Politécnica de Valencia.

${ }^{15}$ Hernández, J. S., Tobón, S., y Vázquez, J. M. (2014). Estudio conceptual de la docencia socioformativa. Ra-Ximhai, 10(5).

${ }^{16}$ De Arteche, M. (2011). Retos y alternativas de la gestión del conocimiento (GC) como propuesta para la colaboración en organizaciones inteligentes. Educar, 47(1).

${ }^{17}$ La ecología del conocimiento-A la ecología del aprendizaje: una metáfora para el aprendizaje organizacional. Módulo: Entorno para capitalizar conocimiento.

http://alondra.udea.edu.co/entornoparacapitalizarconocimiento/co/ENTORNO\%20DE\%20APRENDIZAJE_17.html
} 
mismos, aprendiendo a ser más autónomos tanto a la hora de emitir juicios de valor como de responsabilidad personal. ${ }^{18}$

De Arteche, M: 2011, expresa que "todas las personas, desde su historia personal e intenciones actuales, pueden contribuir al enriquecimiento y a las posibilidades futuras de sus comunidades. Esa contribución debe ser estimulada de manera que las estructuras y prácticas apoyen dicha intención".

El conocimiento social no debe de estar exento de la educación en todas sus etapas y mucho menos de las aulas universitarias porque es ahí donde se forjan, se construye o reconstruye al nuevo ciudadano. La memoria social, la de los pueblos, las comunidades deben de servir de catalizador que armonice los saberes con la complejidad de los contextos de este siglo.

La mayor complejidad en la estructura del conocimiento contemporáneo, que según Edgard Morin solo puede ser asumida por el "pensamiento complejo", impone la interdisciplinariedad como la manera adecuada de dar respuesta a esa complejidad. "La supremacía de un conocimiento fragmentado según las disciplinas, nos dice Morin, impide a menudo operar el vínculo entre las partes y las totalidades y debe dar paso a un modo de conocimiento capaz de aprehender los objetos en sus contextos, sus complejidades, sus conjuntos".

El nuevo paradigma impone a estas organizaciones, desarrollar planes, programas y estructuras curriculares para que los estudiantes desarrollen capacidades científicas para aprender a aprender, construir y resolver los problemas de la diversidad del entorno social y natural. De allí, que ellas no pueden circunscribirse solo a enseñar a usar las nuevas herramientas tecnológicas, sino involucrarse en un programa de desarrollo del conocimiento científico, de la importancia tecnológica y los principios que en ellas se involucran.

De tal forma que el aprendizaje en la educación superior debe de vincularse a la investigación, en ese marco de interdisciplinariedad, cuya premisa aportará con mayores elementos ciertos de juicio para enfrentar o confrontar ese universo que se transforma, se crea y recrea constantemente, lo que produce el criterio que la educación debe ser constante, permanente.

Como expresara Miguel Angel Escotet, "pasar de la idea de una educación terminal a una educación permanente": es decir, el profesional del futuro, estará atrapado de por vida en la educación, y educación y trabajo irán de la mano y no la una a expensas del otro.

La docencia universitaria deberá, entonces, irse ajustada a este escenario que en los países en vías de desarrollo ha sido poco o nada auscultado, lo que ha provocado, a su vez, un abismo no solamente generacional (profesorado y estudiantes) sino también estructural y de comportamiento. Es decir, de contenidos, habilidades y saberes impensados para las generaciones de los siglos anteriores a este.

El cambio de los métodos docentes es una exigencia impuesta por la naturaleza misma del conocimiento contemporáneo, cuyo crecimiento exponencial, multidisciplinariedad e internacionalización, es concomitante de su rápida obsolescencia. Luego, no se trata de una simple innovación pedagógica, sino de algo más profundo: tiene que ver con la estructura misma del conocimiento.

Dice el profesor colombiano Dr. Luis Bernardo Peña: "Un docente que entiende lo que significa esta revolución del conocimiento estará motivado y naturalmente dispuesto a experimentar con nuevas metodologías. Sin ello, los cursos de metodología de la enseñanza o actividades similares no pasarán de tener un carácter "cosmético" y unos efectos transitorios".

En síntesis, y recurriendo a las palabras de la Dra. Inés Aguerrondo: "Un sistema educativo orientado hacia las necesidades del siglo XXI debe incorporar una definición de aprendizaje como el resultado de la construcción activa del sujeto sobre el objeto de aprendizaje. Supone un aprendiz activo, que desarrolla hipótesis propias acerca de cómo funciona el mundo, que deben ser puestas a prueba permanentemente. Supone la generación de operaciones mentales y procedimientos prácticos que permitan seguir aprendiendo solo una vez que se egresó del sistema educativo formal. Supone también que el maestro y el alumno exploran y aprenden juntos, pero que esta exploración y aprendizaje mutuo puede revestir diferentes formas, desde las más presenciales hasta las más remotas"19.

\footnotetext{
${ }^{18}$ Belinchón, R., de Diego, D., y Velasco, M. (2011). Nuevas metodologías docentes aplicadas en el aula. Girona, España: Universitat de Girona.

${ }^{19}$ Gajardo, M. y Puryear, J. (2003). Formas y reformas de la educación en América Latina. (p155)
} 


\section{Preservicio de la educación: las políticas, programas y prácticas en el Ecuador}

En la conferencia mundial sobre la educación superior en el siglo XXI, realizada por la UNESCO, en 1998, en Paris; se destacan aspectos a valorar como considerar a la educación superior como un servicio público.

Que el acceso a la educación superior sea igual para todos. Formar diplomados altamente cualificados y ciudadanos responsables y de constituir un espacio abierto que propicie la formación superior y el aprendizaje a lo largo de toda la vida. Que la pertinencia de la educación superior debe evaluarse en función de la adecuación entre lo que la sociedad espera de las instituciones y lo que éstas hacen. ${ }^{20}$

Antes de referirme a la realidad ecuatoriana es importante señalar datos que provienen del informe de la UNESCO $2013^{21}$, al respecto de la situación educativa de América Latina y el Caribe. La tendencia general del gasto público en educación durante la década pasada fue levemente positiva en la región (pasando aproximadamente de un 4,5\% a un 5,2\% del PIB en promedio), aunque sin un aumento relevante de la priorización de la educación dentro del gasto público. Este mayor gasto parece explicarse principalmente por una expansión del servicio educacional, puesto que el nivel proporcional de gasto público por alumno tendió a mantenerse o a incrementarse levemente en educación primaria y secundaria, y a caer significativamente en educación superior.

El acceso a la educación superior se expandió aceleradamente en la región durante la década del 2000 , acumulando un promedio de crecimiento de aproximadamente $40 \%$, lo que permitió que la región se situara -como conjunto- en el promedio de la tendencia internacional. Aunque la tendencia a la expansión fue muy extendida, persiste entre los países de la región una enorme heterogeneidad en este nivel educativo. El patrón de crecimiento de la educación superior fue, sin embargo, muy inequitativo, favoreciendo principalmente a los sectores de mayores ingresos y de zonas urbanas.

La realidad ecuatoriana guarda sus diferencias frente a los 41 países involucrados en el informe de la UNESCO 2013, debido a sus trascendentales cambios en las políticas, programas y prácticas de las instituciones de educación superior. En Ecuador el gobierno comenzó un proyecto en las políticas educativas post secundaria (2008), con el decreto de la gratuidad de la educación universitaria, el cambio del estatus de los docentes a servidores públicos y la exigencia de un examen actitudinal para los estudiantes del bachillerato. Estrategias que abolieron la autonomía universitaria y el libre ingreso a la misma.

Para el 2010, el gobierno, promulgó la $\mathrm{LOES}^{22}$, con la que creó tres estamentos de control como el Concejo de Educación Superior, CES. Consejo de Evaluación, Acreditación y Aseguramiento de la Calidad de la Educación Superior, CEAACES. Y la Secretaria de Educación Superior, Ciencia, Tecnología e Innovación SENESCYT.

El objetivo de la LOES es regular el Sistema de Educación Superior en el país. La misma garantiza el derecho a la educación superior de calidad, propendiendo a la excelencia, el acceso universal, permanencia, movilidad y egreso sin discriminación. Establece la educación superior como condición indispensable para la construcción del derecho del buen vivir, en el marco de la interculturalidad, respeto a la diversidad y la convivencia armónica con la naturaleza. Garantiza la gratuidad de la educación superior pública hasta el tercer nivel.

Dispone que las instituciones que forman parte del Sistema de Educación Superior rindan cuentas a la sociedad y al Consejo de Educación Superior, sobre el cumplimiento de su misión, fines y objetivos. Establece la igualdad de oportunidades para acceder a becas, créditos y otras formas de apoyo económico. También define ayudas económicas para al menos el 10\% de estudiantes de cada institución.

Crea un sistema de nivelación, para que los alumnos del bachillerato que ingresan con conocimientos desiguales, tengan la oportunidad de equiparar su formación. Además garantiza la educación superior pública gratuita. Obliga a las instituciones de educación superior a someterse a eva-

\footnotetext{
${ }^{20}$ UNESCO. (Octubre de 1998). Conferencia Mundial sobre la Educación Superior. La educación superior en el siglo XXI. Visión y acción. Llevada a cabo en París, Francia.

21 Oficina Regional de Educación para América Latina y el Caribe. (2013). Situación educativa de América Latina y el Caribe. Santiago, Chile: OREALC-UNESCO.

${ }^{22}$ Ley Orgánica de Educación Superior (LOES). Registro Oficial No 298, Quito, Ecuador, 12 de octubre del 2010.
} 
luaciones para el control de la calidad de sus programas y asegura la capacitación permanente y evaluación periódica de los profesores.

Los rectores y vicerrectores serán elegidos por votación de los profesores, estudiantes y trabajadores, y permanecerán en el cargo por cinco años, con una posibilidad de reelección. Determina el principio de cogobierno, que es la dirección compartida de las universidades y escuelas politécnicas, en la que participan profesores, estudiantes, empleados y trabajadores.

Las nuevas políticas de ingreso obligan a los estudiantes secundarios, para ingresar a las universidades, dependiendo de la carrera, a realizar un examen nacional para la educación superior (ENES). Tanto para las carreras de educación como para medicina lo aceptable es un puntaje de 800. Mientras que para las otras carreras es de 700 puntos para su ingreso.

Sim embargo este sistema de ingresar a las universidades públicas en Ecuador no es bien vista por todos, ya que consideran que este examen genera cierto nivel de segmentación social y exclusión. "Es un entrenamiento más que un aprendizaje (...), las pruebas, a la larga, fuerzan para que establecimientos, padres e instituciones induzcan a que repitiendo o entrenando reiteradamente puedan obtener mejores calificaciones", expresa Milton Luna, coordinador del movimiento Contrato Social por la Educación. Añade que el examen nacional de educación superior ENES "no toma en cuenta las inteligencias múltiples o emocionales, solo se evalúan destrezas de lógica formal a través de las matemáticas (modelos) cuestionados".

Por su parte el secretario de la Secretaria de Educación Superior, Ciencia, Tecnología e Innovación SENESCYT, René Ramírez, menciona que: "No podemos tapar el sol con un dedo, existe heterogeneidad en el bachillerato y los estudiantes vienen con diferencias importantes en términos académicos, por eso propusimos en la ley cinco años para que exista una bisagra entre el bachillerato y la universidad, y se den procesos de nivelación y el examen no sea de conocimiento, sino de aptitud". ${ }^{23}$

Ramírez señaló que en este sector el Gobierno invertirá este año 1.656 millones de dólares. Precisó que estos son montos mayores a los destinados por el Gobierno, para la construcción y ampliación de infraestructura vial y a proyectos de electricidad.

Por otro lado el régimen ha creado a través del Consejo de Educación Superior CES cuatro universidades con la finalidad de dar un giro a la matriz productiva del país, generando conocimiento propio, además de mejorar la formación académica del talento humano ecuatoriano.

La Ciudad del Conocimiento Yachay, la Universidad de las Artes, la Universidad del Docente y la Universidad Amazónica Ikiam, especializada en Ciencias de la Vida, son cuatro proyectos ambiciosos del actual Gobierno en el campo de la educación superior.

El costo de este proyecto de creación de estas cuatro universidades es de \$ 1.100 millones de dólares. Lo que para cierto sector universitario esta inversión al igual que los exámenes para ingresar han sido cuestionadas considerando que las universidades públicas también necesitan de recursos económicos para mejorar sus infraestructuras, tecnologías y para capacitación permanente al profesorado con la finalidad de convertirlos en docentes investigadores, para gestionar otros conocimientos.

Dentro del modelo propuesto por el Gobierno Nacional para cambiar la matriz productiva, se plantean numerosos proyectos que contribuyen al desarrollo económico, productivo y social del país. Uno de los elementos estratégicos para este cambio es, sin duda, el sector del conocimiento y talento humano.

Guillaume Long, ministro Coordinador de Conocimiento y Talento Humano (Mccth), manifestó que la transformación de la matriz productiva es un tema que implica un cambio de cimientos estructurales y culturales de la sociedad, por lo que es necesario el conocimiento y talento humano.

"Para cambiar la estructura productiva de un país no se pueden tener solo jornaleros o trabajadores con pocas competencias específicas, sino que se necesita acompañar al nuevo modelo productivo del talento humano del buen vivir", dijo. Agregó que, por ejemplo, para el proceso de industrialización se necesita de una mano de obra altamente calificada y que sea el pilar de ese sector.

\footnotetext{
${ }^{23}$ Ramírez, R. (25 de mayo del 2014). Alto puntaje, condicionante para lograr un cupo de ingreso a las universidades. El Universo.
} 
El titular del Mccth destacó que con estos tres sectores: primario (materias primas con bajo valor agregado), secundario (sector industrial y manufactura) y terciario (servicios), el país contará con una economía más diversificada y para esto se necesita una nueva concepción del talento humano.

Long explicó que esta nueva concepción del talento humano significa impulsar un sector educativo muy fuerte "desde la infancia hasta el doctorado, un sistema integral técnico, tecnólogo y universitario que tenga coherencia, que sea democrático, de calidad y pertinente".

$\mathrm{Al}$ área del conocimiento y talento humano se destinarán alrededor de 800 millones de dólares del Presupuesto General del Estado 2013. ${ }^{24}$

Con todos estos cambios, la práctica docente podría decirse que se está levantando de a poco, pero aún falta mucho por hacer, más que nada en los campos metodológicos-pedagógicos (andragógicos) y en el investigativo, en los que existen altos porcentajes de deficiencias. Dado que muchos de los docentes que trabajan en las universidades, si bien es cierto, son excelente y probados profesionales, pero en el campo de la formación e investigación carecen de recursos cognitivos en estos estadios.

Otro aspecto que deberá ser considerado es, por ejemplo, la evaluación de los aprendizajes, donde justamente esa falta de formación y actualización del profesorado, influyen en los bajos rendimientos cognitivos y por ende del desarrollo de habilidades y destrezas que son el axioma práctico de la enseñanza-aprendizaje.

La formación integral, holística es el camino a seguir en la educación superior en esta sociedad cambiante y compleja de la información y del conocimiento. Pero respetando, así mismo las diferencias, la multiculturalidad, la sostenibilidad y la sustentabilidad de los recursos, el compromiso social para hacer una sociedad más igualitaria, equitativa y de posibilidades para todos.

\section{Conclusiones}

A lo largo de este trabajo teórico se ha intentado analizar el futuro de la universidad ecuatoriana, su vinculación al trabajo, la ciudadanía y la identidad, la enseñanza superior y el aprendizaje, ecologías del conocimiento en cuanto a enlazar la investigación y la docencia en la educación superior, así como las políticas, programas y prácticas, que ha aplicado el Ecuador en comparación de sus pares de América Latina, observando más que nada toda esa carga social y política que intervienen en la educación superior de los diferentes países de la región.

Como vemos son concepciones con múltiples puntos de contacto y que en la actualidad van articulándose bajo las perspectivas de los autores citados y de la realidad presentada en Ecuador en comparación a los países de América Latina.

La universidad en Ecuador y en los países en vías de desarrollo tiene un rol fundamental para cambiar los márgenes de pobreza y miseria, así como hacer a la actual sociedad un espacio con mayores posibilidades de igualdad y equidad entre hombres y mujeres. En base a la aplicación de mejores políticas educativas que permitan el ingreso a más ciudadanos al sistema educativo post secundario. Lo que disminuiría las brechas sociales.

Este enfoque propone visiones diversas en cuanto a los retos de la sociedad de la información y el conocimiento en la gestación de mejores políticas públicas, reestructuras curriculares y pedagógicas de las instituciones de educación superior, de la profesionalización del profesorado y de un entendimiento de la nueva realidad que deberá asumir el ciudadano contemporáneo.

La misma que la podrá enfrentar si tiene herramientas cognitivas, que las comparte con los demás extendiendo redes de conocimientos y en base a ella desarrollar habilidades fundamentadas en valores, lo que vislumbraría pensar en un ser responsablemente social y apto para la convivencia y el mejoramiento de su entorno.

${ }^{24}$ El talento humano aporta a la matriz productiva. (23 de Julio del 2013). El Telégrafo. . 


\section{REFERENCIAS}

Belinchón, R., de Diego, D., y Velasco, M. (2011). Nuevas metodologías docentes aplicadas en el aula. Girona, España: Universitat de Girona.

Comisión Económica para América Latina y el Caribe. (2013). Coyuntura laboral en América Latina y el Caribe. Informe CEPAL/OIT. Santiago, Chile: CEPAL.

Comisión Económica para América Latina y el Caribe. (2014). Panorama Social de América Latina. Santiago, Chile: CEPAL.

UNESCO. (Octubre de 1998). Conferencia Mundial sobre la Educación Superior. La educación superior en el siglo XXI. Visión y acción. Llevada a cabo en París, Francia.

De Arteche, M. (2011). Retos y alternativas de la gestión del conocimiento (GC) como propuesta para la colaboración en organizaciones inteligentes. Educar, 47(1).

Ramírez, R. (25 de mayo del 2014). Alto puntaje, condicionante para lograr un cupo de ingreso a las universidades. El Universo.

Recuperado de: http://www.eluniverso.com/noticias/2014/05/25/nota/3006166/altopuntaje-condicionante-lograr-cupo-ingreso-u

El talento humano aporta a la matriz productiva. (23 de Julio del 2013). El Telégrafo.

Recuperado de: http://voto2013.eltelegrafo.com.ec/economia/item/el-talento-humanoaporta-a-la-matriz-productiva.html

Días, J. (2007). Calidad, pertinencia y responsabilidad social de la universidad latinoamericana y caribeña. En A. L. Gazzola y A. Didriksson (Eds.), Tendencias de la educación superior en América Latina y el Caribe (pp. 87-112). Caracas, Venezuela: UNESCO IESALT.

Fernández, A. (2003). Nuevas metodologías docentes. Valencia, España: Universidad Politécnica de Valencia.

Gajardo, M. e Puryear, J. (2003). Formas y reformas de la educación en América Latina. Santiago de Chile. LOM Ediciones.

Universidad de Antioquía. La ecología del conocimiento-A la ecología del aprendizaje: una metáfora para el aprendizaje organizacional. Módulo: Entorno para capitalizar conocimiento. Recuperado http://alondra.udea.edu.co/entornoparacapitalizarconocimiento/co/ENTORNO\%20DE\%20 APRENDIZAJE_17.html

Ley Orgánica de Educación Superior (LOES). Registro Oficial No 298, Quito, Ecuador, 12 de octubre del 2010.

Mella, E. (2003). La educación en la sociedad del conocimiento y del riesgo. Revista enfoques educacionales, 5(1).

Ministerio de Educación de El Salvador. (2008). Currículo al servicio del aprendizaje. Aprendizaje por competencias. San Salvador, El Salvador: Ministerio de Educación.

Montero, P. (2010). Megatendencias educativas del siglo XX y prospectiva para el siglo XXI: desafios para la educación universitaria. Santiago, Chile: Universidad de Santiago de Chile.

De Alfonseti, N., Lillo, A., Asensi, M. J., Giménez, V. M., Lorenzo, J., Mira-Perceval, M. T., y Rico, J. R. (2007). Metodología docente y sistemas de evaluación del aprendizaje. Alicante, España: Universidad de Alicante.

Pérez, A. (Coord.). (2010). Nuevas exigencias y escenarios para la profesión docente en la era de la información y la incertidumbre. Málaga, España: Universidad de Málaga.

Organización de Estados Iberoamericanos. (2013). El analfabetismo en América Latina. Buenos Aires, Argentina: SITEAL. Recuperado de: http://www.siteal.iipeoei.org/sites/default/files/siteal_datodestacado20130218.pdf

Organización de Estados Iberoamericanos. (2013). ¿Por qué los adolescentes dejan la escuela? Buenos Aires, Argentina: SITEAL. Recuperado de: http://www.siteal.iipeoei.org/sites/default/files/siteal_2013_03_13_dd_28_0.pdf 
Paz y Miño, J. (2008). Universidad ecuatoriana ¿hacia el futuro? Quito, Ecuador: Pontificia Universidad Católica del Ecuador.

Hernández, J. S., Tobón, S., y Vázquez, J. M. (2014). Estudio conceptual de la docencia socioformativa. Ra-Ximhai, 10(5).

Tobón, S. (Julio del 2013). Proyectos formativos: Desarrollo y evaluación de competencias desde la socioformación. En IV Cuarto Congreso Internacional Multidisciplinario. Congreso llevado a cabo en Guadalajara, México.

Comité Científico Regional para América Latina y el Caribe. (2003). Desafíos de la universidad en la sociedad del conocimiento, cinco años después de la Conferencia Mundial sobre Educación Superior. París, Francia: UNESCO.

Oficina Regional de Educación para América Latina y el Caribe. (2013). Situación educativa de América Latina y el Caribe. Santiago, Chile: OREALC-UNESCO.

Universidad Internacional SEK Ecuador. (2011). Modelo educativo basado en competencias de formación integral. Quito, Ecuador: UISEK.

\section{SOBRE EL AUTOR}

César Arturo Carbache Mora: Licenciado en Ciencias de la Comunicación Social, por la Universidad de Guayaquil, con Diplomado Superior en Educación Universitaria por Competencias, por la Universidad del Azuay y Maestría en Educación Superior, por la Universidad Científica del Sur de Perú. Actualmente es docente de la Universidad Laica "Eloy Alfaro" de Manabí, extensión Bahía de Caráquez. Editorialista, radio difusor. Escritor poeta, con publicaciones desde 1993 al 2007, poemarios que han sido presentados en encuentros internacionales de escritores de Perú (2006), México (2011) y Chile (2011 y 2013). Participó en el XXI Congreso de Educación y Aprendizaje, celebrado en Nueva York (2014) en la Universidad Touro - Lander College for Women, donde expuso la ponencia "El aprendizaje en la educación superior". Tiene dos textos inéditos, el uno de corte académico titulado "Una visión a la docencia universitaria en la sociedad de la información y el conocimiento" y el otro un poema intitulado "Sembrada en mis pupilas". Ha dirigido talleres de creación literaria. Promotor cultural independiente. Mentalizador de los seminarios talleres sobre promotores culturales. Ha escrito, dirigido y presentado una serie de obras teatrales. Productor de Proyectos Sociales y Culturales. Mantiene un proyecto en capeta que es la creación de una fundación para el desarrollo cultural. 